A new method for the determination of surface tension from molecular dynamics simulations applied to liquid droplets

This article has been downloaded from IOPscience. Please scroll down to see the full text article.

2011 Chinese Phys. B 20016801

(http://iopscience.iop.org/1674-1056/20/1/016801)

View the table of contents for this issue, or go to the journal homepage for more

Download details:

IP Address: 159.226.231.78

The article was downloaded on 22/03/2012 at 03:30

Please note that terms and conditions apply. 


\title{
A new method for the determination of surface tension from molecular dynamics simulations applied to liquid droplets*
}

\author{
Zhu Ru-Zeng(朱如曾) $)^{\dagger}$ and Yan Hong(间 红) \\ State Key Laboratory of Nonlinear Mechanics (LNM), Institute of Mechanics, \\ Chinese Academy of Sciences, Beijing 100190, China
}

(Received 27 March 2010; revised manuscript received 26 July 2010)

\begin{abstract}
For the determination of surface tension of liquid droplets by molecular dynamics simulations, the most timeconsuming part is the calculation of pressure tensor in the transition layer, which makes it difficult to enhance the precision of the computation. A new method for the calculation of surface tension of liquid droplets to reduce the calculation quantity of pressure tensor in transition layer to the minimum is proposed in this paper. Two thousand particles are taken as example to show how to carry out our scheme.
\end{abstract}

Keywords: surface tension, pressure tensor, molecular dynamics simulation, equimolar surface

PACS: $68.03 . \mathrm{Cd}, 68.35 . \mathrm{Md}$

DOI: $10.1088 / 1674-1056 / 20 / 1 / 016801$

\section{Introduction}

The study of the surface tension of liquids is of great interest to both fundamental and practice, ${ }^{[1]}$ which is increased further especially by the development of nanotechnology. Under nano-scale, the Tolman effect that the surface tension of a liquid droplet changes with the curvature of the surface, ${ }^{[2]}$ is of significance, and so the methods for calculating the surface tension of liquid droplets with different sizes have attracted much research effort. ${ }^{[1-5]}$

For the determination of surface tension of liquid droplets by Molecular Dynamics Simulation (MDS), the most time-consuming part, i.e. the most difficult part to enhance the precision is the calculation of pressure tensor in the surface layer i.e. transition layer. ${ }^{[6]}$ In order to avoid the calculation of pressure tensor in the transition layer, some other kinds of calculation methods were presented in the literatures. A methodology in terms of probability distributions was proposed long time ago by Binder ${ }^{[7]}$ and has received renewed interest recently. ${ }^{[8-10]}$ This method is particularly useful in the vicinity of the critical point. However, the need of sampling all states between the homogeneous bulk phase and the phase coexisting states (slab geometry) makes it fairly time consuming away from the critical point. The method based on the capillary Hamiltonian approximation ${ }^{[8,11,12]}$ is useful to the liquid-vapour interface away from the critical point. Two perturbative approaches have been proposed. One is based on the perturbation of the two-phase system, by increasing or decreasing the interface a finite amount. For curved interfaces, this method resembles the philosophy of Scaled Particle Theory. ${ }^{[13-15]}$ The other is the test area method of Gloor et al. ${ }^{[16,17]}$ MacDowell and Bryk ${ }^{[6]}$ proposed a method which has features of both the Binder method and the perturbative approaches employed by Bresme, Quirke and Gloor et al. Another route is density functional theory. Several density functionals and related self-consistent field theories for polymeric fluids have been proposed in the literatures. ${ }^{[18-21]}$

All of the methods mentioned above belong to approximation theory. In the present paper we propose an accurate method based on rigorous theory. If only the value of surface tension at one radius and one temperature is given, by using our numerical method presented here, we can determine the surface tension of the same substance at any radius and any temperature with no need to calculate the pressure tensor in transition layer. Of course, the given value of surface tension at one radius and one temperature must come from MDS by using the calculation of pressure tensor in the transition layer. However, in our method we can

\footnotetext{
*Project supported by the National Natural Science Foundation of China (Grant No. 10772189) and the Knowledge Innovation Program of Chinese Academy of Sciences.

${ }^{\dagger}$ Corresponding author. E-mail: zhurz@lnm.imech.ac.cn
}

(C) 2011 Chinese Physical Society and IOP Publishing Ltd

http://www.iop.org/journals/cpb http://cpb.iphy.ac.cn 
obtain all the values of surface tensions for all radii and all temperatures at which the liquid droplets can exist, while the calculation of pressure tensor in transition layer is restricted to one radius and one temperature. Thus we do decrease the calculation quantity of pressure tensor in transition layer to the minimum.

Section 2 is devoted to the theoretical basis of our scheme. In Section 3, serving as an illustration, the simulation of 2000 particles has been carried out. The results and some discussions are given in Section 4 and finally, conclusions are drawn in Section 5.

\section{Theoretical basis of our scheme}

For the equimolar surface of a spherical liquid droplet we have

$$
n_{\mathrm{e}}=0
$$

and

$$
F_{\mathrm{e}}=E_{\mathrm{e}}-T S_{\mathrm{e}}=\sigma_{\mathrm{e}} A_{\mathrm{e}},
$$

where $T$ is the temperature of the system, the subscript e denotes the quantity on the equimolar surface, $n_{\mathrm{e}}$ is the number of molecules per unit area of the equimolar surface, $E_{\mathrm{e}}, F_{\mathrm{e}}, S_{\mathrm{e}}, \sigma_{\mathrm{e}}$ and $A_{\mathrm{e}}$ denote the surface energy, surface free energy, surface entropy, surface tension and the area of the equimolar surface, respectively. Then, Eq. (2) gives

$$
\mathrm{d} F_{\mathrm{e}}=\sigma_{\mathrm{e}} \mathrm{d} A_{\mathrm{e}}+A_{\mathrm{e}} \mathrm{d} \sigma_{\mathrm{e}} .
$$

Considering the curvature change, we have ${ }^{[1]}$

$$
\mathrm{d} F_{\mathrm{e}}=-S_{\mathrm{e}} \mathrm{d} T+\sigma_{\mathrm{e}} \mathrm{d} A_{\mathrm{e}}+A_{\mathrm{e}}\left[\frac{\mathrm{d} \sigma}{\mathrm{d} R}\right]_{\mathrm{e}} \mathrm{d} R_{\mathrm{e}}
$$

where $R$ denotes the radius of dividing surface, the derivative $[\mathrm{d} \sigma / \mathrm{d} R]$ is denoted as a notional derivative that is a measure of how the surface tension changes with a change of the position of the dividing surface with the physical state of the system unchanged, $R_{\mathrm{e}}$ denotes the radius of the equimolar surface and is determined by the equation

$$
N=\rho_{\mathrm{l}} \frac{4 \pi}{3} R_{\mathrm{e}}^{3}+\rho_{\mathrm{v}}\left(V-\frac{4 \pi}{3} R_{\mathrm{e}}^{3}\right),
$$

where $N, V, \rho_{\mathrm{l}}$ and $\rho_{\mathrm{v}}$ are the total number of molecules, total volume of the system, density of the interior of the liquid and that of the interior of the vapour, respectively.

Substituting equation (3) into (4) we get

$$
\mathrm{d} \sigma_{\mathrm{e}}\left(T, R_{\mathrm{e}}\right)
$$

$$
=\frac{-S_{\mathrm{e}}\left(T, R_{\mathrm{e}}\right)}{A_{\mathrm{e}}} \mathrm{d} T+\left[\frac{\mathrm{d} \sigma(T, R)}{\mathrm{d} R}\right]_{\mathrm{e}} \mathrm{d} R_{\mathrm{e}}
$$

where $\sigma_{\mathrm{e}}\left(T, R_{\mathrm{e}}\right)$ can also be expressed by $\sigma\left(T, R_{\mathrm{e}}\right)$. Equation (6) determines the way how the differential of surface tension $\mathrm{d} \sigma_{\mathrm{e}}\left(T, R_{\mathrm{e}}\right)$ depends on the two variables $\left(T, R_{\mathrm{e}}\right)$ and their differentials $\left(\mathrm{d} T, \mathrm{~d} R_{\mathrm{e}}\right)$.

In Eq. (6), the surface tension $\sigma_{\mathrm{e}}\left(T, R_{\mathrm{e}}\right)$ is a function of two self variables $\left(T, R_{\mathrm{e}}\right)$, and so the differential $\mathrm{d} \sigma_{\mathrm{e}}\left(T, R_{\mathrm{e}}\right)$ expressed by Eq. (6) is a total differential. Therefore the integral

$$
\begin{aligned}
& \int_{\left(T_{0}, R_{\mathrm{e} 0}\right)}^{\left(T_{1}, R_{\mathrm{e} 1}\right)} \mathrm{d} \sigma_{\mathrm{e}}\left(T, R_{\mathrm{e}}\right) \\
= & \int_{\left(T_{0}, R_{\mathrm{e} 0}\right)}^{\left(T_{1}, R_{\mathrm{e} 1}\right)}\left\{\frac{-S_{\mathrm{e}}\left(T, R_{\mathrm{e}}\right)}{A_{\mathrm{e}}} \mathrm{d} T+\left[\frac{\mathrm{d} \sigma(T, R)}{\mathrm{d} R}\right]_{\mathrm{e}} \mathrm{d} R_{\mathrm{e}}\right\}
\end{aligned}
$$

must be determined by the origin point and end point, and be independent of the path.

Below we discuss two different paths, i.e. restrictive conditions in the $T R_{\mathrm{e}}$ plane

$$
R_{\mathrm{e}}=R_{\mathrm{e}}(T)
$$

and

$$
T=T_{0}
$$

respectively, where $R_{\mathrm{e}}(T)$ and $T_{0}$ may be appointed arbitrarily.

\subsection{Under the restrictive conditions $R_{\mathrm{e}}=R_{\mathrm{e}}(T)$}

Obviously, for any given restrictive relation $R_{\mathrm{e}}=$ $R_{\mathrm{e}}(T)$, there must be an infinite set of liquid droplets satisfying this relation. For this set of liquid droplets, the surface tension $\sigma_{\mathrm{e}}$ and entropy $S_{\mathrm{e}}$ depend only on one variable $T$ and can be expressed as $\sigma_{\mathrm{e}}(T)=\sigma_{\mathrm{e}}\left(T, R_{\mathrm{e}}(T)\right)=\sigma\left(T, R_{\mathrm{e}}(T)\right)$ and $S_{\mathrm{e}}(T)=$ $S_{\mathrm{e}}\left(T, R_{\mathrm{e}}(T)\right)$, respectively. Therefore Eq. (6) becomes the monadic differential form

$$
\begin{aligned}
& \mathrm{d} \sigma_{\mathrm{e}}\left(T, R_{\mathrm{e}}(T)\right) \\
= & \frac{-S_{\mathrm{e}}\left(T, R_{\mathrm{e}}(T)\right)}{A_{\mathrm{e}}} \mathrm{d} T+\left[\frac{\mathrm{d} \sigma(T, R)}{\mathrm{d} R}\right]_{\mathrm{e}}\left(\frac{\mathrm{d} R_{\mathrm{e}}(T)}{\mathrm{d} T}\right) \mathrm{d} T,
\end{aligned}
$$

which gives

$$
\begin{aligned}
& \frac{\mathrm{d} \sigma_{\mathrm{e}}\left(T, R_{\mathrm{e}}(T)\right)}{\mathrm{d} T} \\
= & \frac{-S_{\mathrm{e}}\left(T, R_{\mathrm{e}}(T)\right)}{A_{\mathrm{e}}}+\left[\frac{\mathrm{d} \sigma(T, R)}{\mathrm{d} R}\right]_{\mathrm{e}}\left(\frac{\mathrm{d} R_{\mathrm{e}}(T)}{\mathrm{d} T}\right) .
\end{aligned}
$$

From equations (2) and (9), we obtain 


$$
\frac{\mathrm{d} \sigma_{\mathrm{e}}\left(T, R_{\mathrm{e}}(T)\right)}{\mathrm{d} T}=\frac{\sigma_{\mathrm{e}}\left(T, R_{\mathrm{e}}(T)\right)}{T}-\frac{\varepsilon_{\mathrm{e}}\left(T, R_{\mathrm{e}}(T)\right)}{T}+\left[\frac{\mathrm{d} \sigma(T, R)}{\mathrm{d} R}\right]_{\mathrm{e}} \frac{\mathrm{d} R_{\mathrm{e}}(T)}{\mathrm{d} T},
$$

where $\varepsilon_{\mathrm{e}}$ is the energy per unit area of the equimolar surface. Equation (10) with the general Laplace equation ${ }^{[1]}$

$$
\Delta p=p_{1}-p_{\mathrm{v}}=\frac{2 \sigma_{\mathrm{e}}}{R_{\mathrm{e}}}+\left[\frac{\mathrm{d} \sigma}{\mathrm{d} R}\right]_{\mathrm{e}}
$$

gives

$$
\frac{\mathrm{d} \sigma_{\mathrm{e}}\left(T, R_{\mathrm{e}}(T)\right)}{\mathrm{d} T}+\left(T \frac{\mathrm{d} R_{\mathrm{e}}(T)}{\mathrm{d} T} \frac{2}{R_{\mathrm{e}}}-1\right) \frac{\sigma_{\mathrm{e}}\left(T, R_{\mathrm{e}}(T)\right)}{T}=\Delta p\left(T, R_{\mathrm{e}}(T)\right) \frac{\mathrm{d} R_{\mathrm{e}}(T)}{\mathrm{d} T}-\frac{\varepsilon_{\mathrm{e}}\left(T, R_{\mathrm{e}}(T)\right)}{T},
$$

where $p_{1}$ and $p_{\mathrm{v}}$ denote the pressures in the interior of the liquid and in the interior of the vapour respectively.

If for a liquid, we give a function $R_{\mathrm{e}}(T)$ arbitrarily and know the functions $\varepsilon_{\mathrm{e}}(T)=\varepsilon_{\mathrm{e}}\left(T, R_{\mathrm{e}}(T)\right)$ and $\Delta p(T)=\Delta p\left(T, R_{\mathrm{e}}(T)\right)$, then Eq. (12) is a first-order ordinary differential equation for function $\sigma_{\mathrm{e}}(T)=$ $\sigma_{\mathrm{e}}\left(T, R_{\mathrm{e}}(T)\right)$. The solution of Eq. (12) is

$$
\begin{aligned}
\sigma_{\mathrm{e}}(T)= & \sigma_{\mathrm{e}}\left(T, R_{\mathrm{e}}(T)\right) \\
= & \mathrm{e}^{-\int_{T_{0}}^{T}\left(T^{\prime} \frac{\mathrm{d} R_{\mathrm{e}}}{\mathrm{d} T^{\prime}} \frac{2}{R_{\mathrm{e}}}-1\right) \frac{\mathrm{d} T^{\prime}}{T^{\prime}}}\left(\int_{T_{0}}^{T}\left(\Delta p \frac{\mathrm{d} R_{\mathrm{e}}}{\mathrm{d} T^{\prime}}-\frac{\varepsilon_{\mathrm{e}}}{T^{\prime}}\right) \mathrm{e}^{\int_{T_{0}}^{T^{\prime}}\left(T^{\prime \prime} \frac{\mathrm{d} R_{\mathrm{e}}}{\mathrm{d} T^{\prime \prime}} \frac{2}{R_{\mathrm{e}}}-1\right) \frac{\mathrm{d} T^{\prime \prime}}{T^{\prime}}} \mathrm{d} T^{\prime}+\sigma_{\mathrm{e}}\left(T_{0}, R_{\mathrm{e}}\left(T_{0}\right)\right)\right) \\
= & \frac{T}{3 R_{\mathrm{e}}^{2}(T)}\left(\left.R_{\mathrm{e}}^{3}\left(T^{\prime}\right) \frac{\Delta p\left(T^{\prime}\right)}{T^{\prime}}\right|_{T_{0}} ^{T}-\int_{T_{0}}^{T} R_{\mathrm{e}}^{3}\left(T^{\prime}\right) \frac{T^{\prime} \frac{\mathrm{d} \Delta p}{\mathrm{~d} T^{\prime}}-\Delta p\left(T^{\prime}\right)}{T^{\prime 2}} \mathrm{~d} T^{\prime}\right) \\
& -\frac{T}{R_{\mathrm{e}}^{2}(T)} \int_{T_{0}}^{T} \frac{\varepsilon_{\mathrm{e}}\left(T^{\prime}\right) R_{\mathrm{e}}^{2}\left(T^{\prime}\right)}{T^{\prime 2}} \mathrm{~d} T^{\prime}+\sigma_{\mathrm{e}}\left(T_{0}, R_{\mathrm{e}}\left(T_{0}\right)\right) \frac{T R_{\mathrm{e}}^{2}\left(T_{0}\right)}{T_{0} R_{\mathrm{e}}^{2}(T)} .
\end{aligned}
$$

Using formula (13) we can calculate function $\sigma_{\mathrm{e}}(T)=$ $\sigma_{\mathrm{e}}\left(T, R_{\mathrm{e}}(T)\right)$ in any given interval $\left(T_{0}, T_{1} ; T_{0} \neq T_{1}\right)$ for a given single component liquid and a given function $R_{\mathrm{e}}(T)$ in interval $\left(T_{0}, T_{1}\right)$, if the value $\sigma_{\mathrm{e}}\left(T_{0}\right)$ is known, and functions $\Delta p(T)$ and $\varepsilon_{\mathrm{e}}(T)$ in interval $\left(T_{0}, T_{1}\right)$ are also known. In fact, the $\sigma_{\mathrm{e}}\left(T_{0}\right)$ can be obtained through experimental measurement or by calculation with the MDS results of pressure tensors in the transition layer, shown in Subsection 1.3. below; the function $\Delta p(T)$ can be calculated by MDS in the interiors of the liquid droplet and the vapour; and the function

$$
\begin{aligned}
& \varepsilon_{\mathrm{e}}(T) \\
= & \frac{1}{4 \pi R_{\mathrm{e}}}\left(E-\frac{4 \pi}{3} R_{\mathrm{e}}^{3} \varepsilon_{1}-\left(V-\frac{4 \pi}{3} R_{\mathrm{e}}^{3}\right) \varepsilon_{\mathrm{v}}\right)
\end{aligned}
$$

can be calculated by MDS with Eq. (5), where $E, \varepsilon_{1}$ and $\varepsilon_{\mathrm{v}}$ are the total energy, energy densities of the interior of the liquid and the vapour, respectively. And so we need not use the pressure tensor in surface layer. Besides, to calculate $E, \varepsilon_{1}$ and $\varepsilon_{\mathrm{v}}$ in Eq. (14), we must use the energy of a molecule

$$
E_{\mathrm{m}}=\frac{m}{2} v^{2}+\frac{U}{2}
$$

where $m, v, U$ are molecular mass, molecular speed and inter-molecule potential respectively.

If we want to calculate $\sigma_{\mathrm{e}}\left(T_{2}, R_{\mathrm{e} 2}\right)$ from $\sigma_{\mathrm{e}}\left(T_{0}, R_{\mathrm{e} 0}\right)$ by Eq. (13), in which $T_{0}, T_{2}, R_{\mathrm{e} 0}$ and $R_{\mathrm{e} 2}$ are appointed arbitrarily, first we should make a arbitrary function $R_{\mathrm{e}}(T)$ satisfying the conditions $R_{\mathrm{e}}\left(T_{2}\right)=R_{\mathrm{e} 2}$ and $R_{\mathrm{e}}\left(T_{0}\right)=R_{\mathrm{e} 0}$, then to calculate it according to the above method. Therefore the restrictive condition is only a tool to transfer the Eq. (6) of two self-variables to Eq. (9) of single self-variable. Due to the arbitrariness of the function $R_{\mathrm{e}}(T)$, there is no obstacle for us to use this method to calculate $\sigma_{\mathrm{e}}\left(T, R_{\mathrm{e}}\right)$ for any pair of $\left(T, R_{\mathrm{e}}\right)$ from a known $\sigma_{\mathrm{e}}\left(T_{0}, R_{\mathrm{e} 0}\right)$, if only there does exist a liquid droplet at $\left(T, R_{\mathrm{e}}\right)$. 


\subsection{Under the restrictive conditions $T=T_{0}$}

$T=T_{0}$ is a special condition, which can not be expressed by the type of restrictive condition $R_{\mathrm{e}}(T)$. For any given temperature $T_{0}$, the restrictive relation $T=T_{0}$ in $T R_{\mathrm{e}}$ plane corresponds to a infinite set of liquid droplets satisfying this isothermal condition. For this set of liquid droplets, the surface tension $\sigma_{\mathrm{e}}$ and surface entropy $S_{\mathrm{e}}$ depend only on one variable $R_{\mathrm{e}}$ and can be expressed as $\sigma_{\mathrm{e}}\left(R_{\mathrm{e}}\right)=\sigma_{\mathrm{e}}\left(T_{0}, R_{\mathrm{e}}\right)$ and $S_{\mathrm{e}}\left(R_{\mathrm{e}}\right)=S_{\mathrm{e}}\left(T_{0}, R_{\mathrm{e}}\right)$, respectively. Therefore Eq. (5) becomes the monadic differential form

$$
\left[\frac{\mathrm{d} \sigma}{\mathrm{d} R}\right]_{\mathrm{e}} \mathrm{d} R_{\mathrm{e}}=\mathrm{d} \sigma_{\mathrm{e}}\left(T_{0}, R_{\mathrm{e}}\right) .
$$

Combination of Eq. (16) and general Laplace equation (11) gives

$$
\frac{\mathrm{d} \sigma_{\mathrm{e}}\left(R_{\mathrm{e}}, T_{0}\right)}{\mathrm{d} R_{\mathrm{e}}}+\frac{2}{R_{\mathrm{e}}} \sigma_{\mathrm{e}}\left(R_{\mathrm{e}}, T_{0}\right)=\Delta p\left(R_{\mathrm{e}}, T_{0}\right) .
$$

Under the condition

$$
\sigma\left(R_{\mathrm{e} 0}, T_{0}\right)=\sigma_{0}
$$

the solution of Eq. (17) is

$$
\begin{aligned}
& \sigma_{\mathrm{e}}\left(R_{\mathrm{e}}, T_{0}\right) \\
= & \sigma\left(R_{\mathrm{e}}, T_{0}\right) \frac{1}{R_{\mathrm{e}}^{2}}\left[R_{\mathrm{e} 0}^{2} \sigma_{0}+\int_{R_{\mathrm{e} 0}}^{R_{\mathrm{e}}} R^{2} \Delta p \mathrm{~d} R\right] .
\end{aligned}
$$

\subsection{A method for calculation of initial value $\sigma_{\mathrm{e} 0}=\sigma\left(T_{0}, R_{\mathrm{e} 0}\right)$ by $\mathrm{MDS}$}

Now we show how to obtain an initial value $\sigma_{\mathrm{e} 0}=$ $\sigma\left(T_{0}, R_{\mathrm{e} 0}\right)$ by MDS for lack of experiment data. For a surface of tension with radius $R_{\mathrm{s}}$, we have Laplace equation

$$
R_{\mathrm{s}}=\frac{2 \sigma_{\mathrm{s}}}{p_{1}-p_{\mathrm{v}}}
$$

And ${ }^{[22]}$

$$
\sigma_{\mathrm{s}}^{3}=-\frac{1}{8}\left(p_{\mathrm{l}}-p_{\mathrm{v}}\right)^{3} \int_{0}^{R_{\infty}} r^{3} \frac{\mathrm{d} p_{\mathrm{N}}(r)}{\mathrm{d} r} \mathrm{~d} r,
$$

where $R_{\infty}$ is a $r$ value of any point in the interior of the vapour and $p_{\mathrm{N}}(r)$ is the normal pressure

$$
p_{\mathrm{N}}(r)=p_{\mathrm{K}}(r)+p_{\mathrm{U}}(r),
$$

where $p_{\mathrm{U}}(r)$ is configurational normal pressure, and $p_{\mathrm{K}}(r)$ is kinetic pressure

$$
p_{\mathrm{K}}(r)=k_{\mathrm{B}} T n(r)
$$

with $k_{\mathrm{B}}$ and $n(r)$ being Boltzmann constant and the density of the number of molecules, respectively.

Integrating the right of Eq. (21) by parts gives

$$
\begin{aligned}
\sigma_{\mathrm{s}}^{3} & -\frac{1}{8}\left(p_{\mathrm{l}}-p_{\mathrm{v}}\right)^{2} \\
& \times\left\{R_{\infty}^{3} p_{\mathrm{N}}\left(R_{\infty}\right)-3 \int_{0}^{R_{\infty}} r^{2} p_{\mathrm{N}}(r) \mathrm{d} r\right\} .
\end{aligned}
$$

The Irving-Kirkwood definition for pressure tension has been accepted commonly as the correct formula. ${ }^{[23]}$

In order to use MDS for the calculation of Eqs. (24) and (20) with Irving-Kirkwood definition, we use the symbols of Thompson ${ }^{[22]}$ and have

$$
p_{\mathrm{U}}(r)=S^{-1} \sum_{k} f_{k},
$$

where $S=4 \pi r^{2}$ is the area of a spherical dividing surface of radius $r$ with its centre being mass centre of the liquid, and the sum over $k$ is over the normal components $f_{k}$ of all the pair forces acting across the surface. ${ }^{[22]}$ For any given radius $r$ between 0 and $R_{\infty}$, the values of $p_{\mathrm{U}}(r)$ and $p_{\mathrm{K}}(r)$ can be calculated by Eqs. (25) and (23) with MDS under given $V, N$ and $T_{0}$, respectively. Substituting the values of $p_{\mathrm{U}}(r)$ and $p_{\mathrm{K}}(r)$ into Eq. $(22)$ gives $p_{\mathrm{N}}(r)$. Thus we can obtain numerical functions $p_{\mathrm{N}}(r)$. Then we obtain the surface tension of the surface of tension $\sigma_{\mathrm{s}}=\sigma_{s 0}$ by substituting the numerical functions $p_{\mathrm{N}}(r)$ into Eq. (24) and integrating it. Then substituting the values of $\left(p_{1}-p_{\mathrm{v}}\right)$ given by MDS and $\sigma_{\mathrm{s} 0}$ into the Laplace equation (20) gives the radius of the surface of tension $R_{\mathrm{s}}=R_{\mathrm{s} 0}$. The radius of equimolar surface $R_{\mathrm{e} 0}$ can easily be determined by using Eq. (5) and MDS. Then the relation ${ }^{[1]}$

$$
\frac{\sigma_{\mathrm{e} 0}}{\sigma_{\mathrm{s} 0}}=1+\left(\frac{R_{\mathrm{e} 0}-R_{\mathrm{s} 0}}{R_{\mathrm{e} 0}}\right)^{2} \frac{\left(R_{\mathrm{s} 0}+2 R_{\mathrm{e} 0}\right)}{3 R_{\mathrm{s} 0}}
$$

gives the $\sigma_{\mathrm{e} 0}$, which is just what we want $\sigma_{\mathrm{e} 0}=$ $\sigma_{\mathrm{e}}\left(T_{0}, R_{\mathrm{e} 0}\right)$.

\section{Computer simulation}

Serving as an illustration of our scheme described above, we take 2000 particles to carry out this scheme for restrictive conditions $R_{\mathrm{e}}=R_{\mathrm{e}}(T)$.

The Lennard-Jones potential between particles has the form

$$
U(r)=4 \varepsilon\left\{\left(\frac{d}{r}\right)^{12}-\left(\frac{d}{r}\right)^{6}\right\},
$$


where $r, \varepsilon$ and $d$ are the inter-particle distance, energy scale and length scale respectively. The parameters are chosen as $\varepsilon / k_{\mathrm{B}}=93.16, d=0.3405 \mathrm{~nm}$ and $r_{\text {cut }}=3.6 d$, where $r_{\text {cut }}$ is the cutoff distance.

The system of $N=2000$ particles investigated is a spherical droplet being equilibrated with its vapour enclosed in a cubic box with a size of $x \times y \times z=$ $28.0 d \times 28.0 d \times 28.0 d$. The initial configuration is constructed by putting particles on a finite cubic lattice located at the central part of the box. The mirror boundary condition is used in all directions.

At the initial time the velocities of particles were given according to the Maxwell-Boltzmann distribution of $T_{0}=66 \mathrm{~K}$. NVT ensemble was used before equilibration. To reach equilibrium we did up to 100000 runs with a time step of $\delta t=5$ fs. For calculating the mean value of any physical quantity $g(t)$, accumulative average $\overline{g(i \cdot \delta t)}=(1 / M) \sum_{i=1}^{M} g(i \cdot \delta t)$ was used, where the number $M$ must be large enough for the accumulative mean value to reach a constant with acceptable small variation. In fact in our simulations up to 1000000 runs with a time step of $\delta t=2 \mathrm{fs}$ were used to obtain the acceptable statistics.

The values of $R_{\mathrm{e} 0}=R_{\mathrm{e}}\left(T_{0}\right)$ and $\sigma_{\mathrm{e} 0}=$ $\sigma_{\mathrm{e}}\left(T_{0}, R_{\mathrm{e} 0}\right)$ were calculated by MDS with the method given in Subsection 1.3. In this respect, for the numerical functions $p_{\mathrm{U}}(r)$ and $p_{\mathrm{K}}(r)$, we took fifty values of $r$ with equal spacing.

In this study our aim of the stimulation is only to give a simple example for our method, therefore to avoid the expensive costs of computational time for adjustment of the particle number of the system according to a given function $R_{\mathrm{e}}(T)$, we changed the temperature $T$ and kept the total number of particles of the system including liquid and vapour $N$ unchanged, so that the equimolar radius $R_{\mathrm{e}}$ changed with the temperature spontaneously. Thus we easily obtained a numerical function $R_{\mathrm{e}}(T)$ given by MDS. In fact, we heated the system gradually from $66 \mathrm{~K}$ to $86 \mathrm{~K}$ with an interval of $2 \mathrm{~K}$.

The numerical function $\Delta p(T)$ was calculated for every $2 \mathrm{~K}$ by Eqs. (22), (23), (25) and MDS.

The numerical function $\varepsilon_{\mathrm{e}}(T)$ was calculated by Eqs. (14), (15), (27) and MDS.

The numerical function $\sigma_{\mathrm{e}}(T)=\sigma\left(T, R_{\mathrm{e}}(T)\right)$ was calculated by Eq. (13) with use of $R_{\mathrm{e} 0}$ and $\sigma_{\mathrm{e} 0}$.

In this simulation, all the quantities were made dimensionless in terms of the Lennard-Jones parameters, i.e., $r^{*}=r / d, T^{*}=k_{\mathrm{B}} T / \varepsilon, \rho^{*}=\rho d^{3}, p^{*}=$ $p d^{3} / \varepsilon, \delta t^{*}=\delta t \sqrt{\left(\varepsilon / m d^{2}\right)}$, by which $5 \mathrm{fs}$ is equal to
0.002319 dimensionless time and $m$ is the molecular mass of Argon.

\section{Results and discussion}

For the system of 2000 particles in a cubic box with a size of $28.0 d \times 28.0 d \times 28.0 d$ at $T=T_{0}=$ $66 \mathrm{~K}$, we obtain $\sigma_{\mathrm{s}}^{*}=\sigma_{s 0}^{*}=0.7901, \quad R_{\mathrm{s}}^{*}=R_{s 0}^{*}=$ $7.8350, \quad R_{\mathrm{e}}^{*}=R_{\mathrm{e} 0}^{*}=8.2687$, and $\sigma_{\mathrm{e}}^{*}=\sigma_{\mathrm{e} 0}^{*}=0.7925$. The normal component profile of pressure tensor used to calculate $\sigma_{s 0}^{*}$ by Eq. (24) is shown in Fig. 1, and the number density profile used to calculate $R_{\mathrm{e} 0}^{*}$ is shown in Fig. 2. The big fluctuation of $p_{\mathrm{N}}^{*}$ near the centre of the liquid in Fig. 1 attributes to the poor statistics because of the low volume.

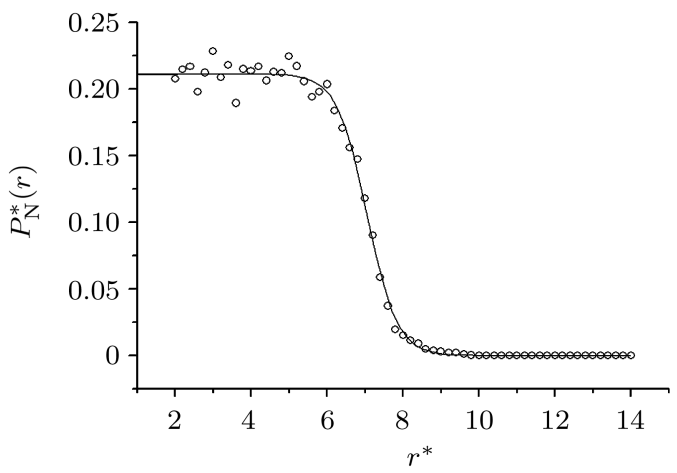

Fig. 1. Normal component of the IK pressure tensor at $T=66 \mathrm{~K}$.

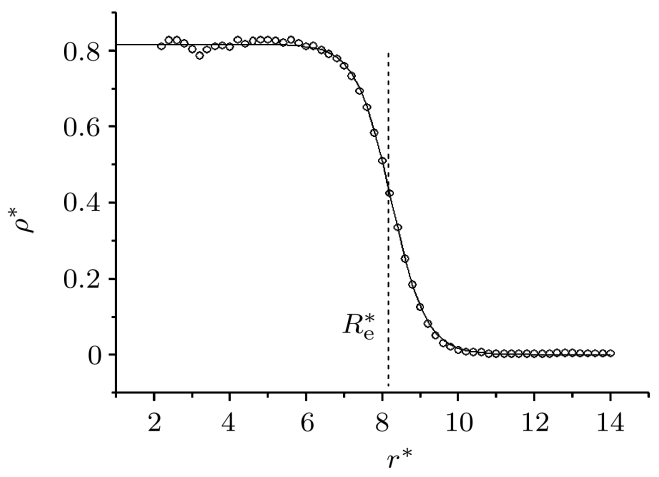

Fig. 2. The density profile at $T=66 \mathrm{~K}$. The dashed line indicates the radius of equimolar surface.

The results of numerical functions $R_{\mathrm{e}}^{*}(T)$, $\Delta p^{*}(T), \varepsilon_{\mathrm{e}}^{*}(T)$ and $\sigma_{\mathrm{e}}^{*}(T)$ for $N=2000$ are shown in Table 1, and the calculated temperature dependence of the surface tension $\sigma_{\mathrm{e}}^{*}-T^{*}$ for $N=2000$ is shown in Fig. 3. From Table 1, we know that the $R_{\mathrm{e}}^{*}$ decreases with increasing temperature, which is caused by evaporation. As can be seen from Fig. 3, there is an overall decrease in the magnitude of the surface tension 
with increasing temperature, which is in good qualitative agreement with the predictions of thermodynamical theories. ${ }^{[22]}$ More exactly, $\sigma_{\mathrm{e}}^{*}$ decreasing with increasing temperature attributes not only to the temperature change itself but also to the decrease of $R_{\mathrm{e}}^{*}$, which is called Tolman effect. ${ }^{[2]}$ The behaviour of $\sigma_{\mathrm{e}}^{*}$ attributes to both of Tolman effect and temperature effect.

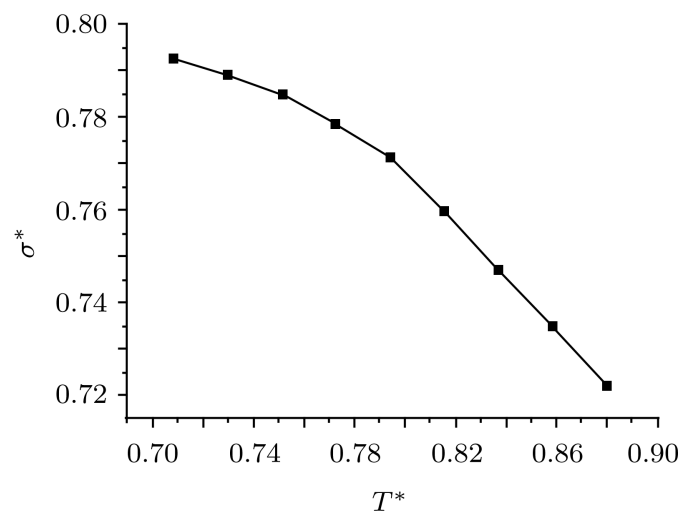

Fig. 3. The plot of $\sigma_{\mathrm{e}}^{*}-T^{*}$ for $N=2000$.

Table 1. The MDS results of surface tension and related intermediate results.

\begin{tabular}{cccccc}
\hline$T$ & $T^{*}$ & $R_{\mathrm{e}}^{*}$ & $\Delta p^{*}$ & $\varepsilon_{\mathrm{e}}^{*}$ & $\sigma_{\mathrm{e}}^{*}$ \\
\hline 66 & 0.70846 & 8.2687 & 0.2011 & -0.0209 & 0.7925 \\
68 & 0.72993 & 8.2551 & 0.1950 & 0.0130 & 0.7891 \\
70 & 0.75140 & 8.2367 & 0.1885 & 0.0400 & 0.7848 \\
72 & 0.77286 & 8.2120 & 0.1800 & 0.0760 & 0.7785 \\
74 & 0.79433 & 8.1857 & 0.1718 & 0.1099 & 0.7712 \\
76 & 0.81580 & 8.1393 & 0.1610 & 0.1680 & 0.7596 \\
78 & 0.83727 & 8.0910 & 0.1452 & 0.2226 & 0.7470 \\
80 & 0.85874 & 8.0456 & 0.1230 & 0.2550 & 0.7348 \\
82 & 0.88021 & 7.9875 & 0.1060 & 0.2920 & 0.7219 \\
\hline
\end{tabular}

Besides, We note that the experimental surface tension value of a planar liquid-argon surface at $90 \mathrm{~K}$ $\left(T^{*}=0.97\right)$ is $13.45 \mathrm{mN} / \mathrm{m}^{[5]}\left(\sigma_{\infty}^{*}=1.08\right)$, which is bigger than our result $\sigma_{\infty}^{*}=0.67$ (for $90 \mathrm{~K}, R_{\mathrm{e}}^{*}=7.79$ ) extrapolated by the data in Table 1 . These are coincident with Tolman formula ${ }^{[23]}$

$$
\sigma^{*}\left(R_{\mathrm{e}}\right)=\frac{\sigma_{\infty}^{*}}{1+\left(2 \delta^{*} / R_{\mathrm{e}}^{*}\right)}
$$

with $\delta^{*}=2.38$, i.e. $\delta=2.38 d$. We know that in the Table 3 of Ref. [22], there are four computational data of Tolman length $\delta^{*}: 1.11,0.96,2.7$ and 4.04 for $N=2048$ and $T^{*} \approx 0.70$. Their average value is $\delta^{*}=2.20$, which is coincident with that in Eq.(28), though the two temperatures are different. If we notice that 'Despite increasing theoretical attention, debate continues on even the sign of Tolman's length for simple liquids different' ${ }^{\text {'24] }}$ and 'Recent MD simulations furthermore indicate that the Tolman length sensitively depends on the interaction potential. ${ }^{\text {[24] }}$ Our agreement is satisfactory.

All the analysis in this section shows that our theoretical scheme is a good or successful attempt and worthy of studying.

\section{Conclusions}

For the determination of surface tension of liquid droplets from molecular dynamics simulations, the most time-consuming part, i.e. the most difficult part to enhance the precision, is the calculation of pressure tensors in the surface layer i.e. transition layer. To overcome this difficulty, we have deduced a formula that permits us to calculate the surface tensions by MDS for all temperatures and all radii at which the liquid droplets can exist from the surface tension of a given temperature and a given radius. The advantage of this method consists in decreasing the calculation quantity of pressure tensors in transition layer to a minimum. Two thousand particles are taken to show how to carry out our scheme.

\section{References}

[1] Rowlinson J S and Widom B 1982 Molecular Theory of Capillary Clarendon Press (New York: Oxford University Press)

[2] Tolman R C 1949 J. Chem. Phys. 17333

[3] Irving J H and Kirkwood J G 1950 J. Chem. Phys. 1817

[4] Magda J J, Tirrell M and Davis H T 1985 J. Chem. Phys. 831888
[5] Croxton C A 1980 Statistical Mechanics of the Liquid State. (Bath: John Wiley Press)

[6] MacDowel L G and Bryk P 2007 Phys. Rev. E 75061609

[7] Binder K 1982 Phys. Rev. A 251699

[8] Müller M and MacDowel L G 2000 Macromolecules 33 3902

[9] Potoff J J and Panagiotopoulos A Z 2000 J. Chem. Phys. 1126411

[10] Errington J R 2003 Phys. Rev. E 67012102 
[11] Milchev A and Binder K 2001 J. Chem. Phys. 1148610 [12] Müller M and Schick M 1996 J. Chem. Phys. 1058282

[13] Reiss H, Frisch H L and Lebowitz J L 1959 J. Chem. Phys. 31369

[14] Henderson J R 1983 Mol. Phys. 50741

[15] Bryk P, Roth R, Mecke K R and Dietrich S 2003 Phys. Rev. E 68031602

[16] Gloor G J, Jackson G, Blas F J and de Miguel E $2005 \mathrm{~J}$. Chem. Phys. 123134703

[17] de Miguel E and Jackson G 2006 Mol. Phys. 1043717

[18] Woodward C E 1990 J. Chem. Phys. 943183
[19] Chandler D, McCoy J D and Singer S J 1986 J. Chem. Phys. 855971

[20] Helfand E 1972 J. Chem. Phys. 563592

[21] Müller M and Macdowel L G 2003 J. Phys.: Condens. Matter 15 R609

[22] Thompson S M, Gubbins K E, Walton J P R B, Chantry R A R and Rowlinson J S 1984 J. Chem. Phys. 81530

[23] Vrabec J, Kedia G K, Fuchs G and Hasse H 2006 Mol. Phys. 1041509

[24] Blokhuis E M and Kuipers J 2006 J.Chem. Phys. 124 074701 